Supporting information

\title{
Infrared Spectrum of the Amantadine Cation: \\ Opening of the Diamondoid Cage upon lonization
}

\author{
Martin Andreas Robert George and Otto Dopfer*
}

Prof. Dr. Otto Dopfer and M. A. R. George (MSc)

Institut für Optik und Atomare Physik, Technische Universität Berlin, Hardenbergstr. 36, 10623 Berlin (Germany)

* corresponding author; email: dopfer@physik.tu-berlin.de; ORCID: 0000-0002-9834-4404 


\section{Table of Contents}

\section{Experimental and Theoretical Methods}

Table S1. Computed vibrational frequencies (in $\mathrm{cm}^{-1}$, B3LYP-D3/cc-pVTZ) of $\mathrm{Ama}^{+}(\mathrm{I}-\mathrm{III})$ compared to experimental values of $\mathrm{Ama}^{+} \mathrm{Ar}$ (Figure 1).

Table S2. Various energies (in $\mathrm{kJmol}^{-1}$ ) of $\mathrm{Ama}^{+}(\mathrm{I}-\mathrm{III}), \mathrm{Ama}^{+}(\mathrm{I}-\mathrm{III})-\mathrm{Ar}$ and $\mathrm{Ada}^{+}(\mathrm{I}-\mathrm{III})$ isomers calculated at the B3LYP-D3/cc-pVTZ level.

Table S3. Various energies (in $\mathrm{kJmol}^{-1}$ ) of $\mathrm{Ama}^{+}(\mathrm{I}-\mathrm{III})-\mathrm{Ar}(\mathrm{I}-\mathrm{VIII})$ isomers calculated at the B3LYP-D3/cc-pVTZ level.

Figure S1. Calculated equilibrium structures (in $\AA$ and degrees) of $\mathrm{Ama}^{+}(\mathrm{I}-\mathrm{III})$ isomers compared to their most stable Ama ${ }^{+}(\mathrm{I}-\mathrm{III})$-Ar isomers calculated at the B3LYP-D3/cc-pVTZ level.

Figure S2. NBO charge distribution (in me) of $\mathrm{Ama}^{+}(\mathrm{I}-\mathrm{III})$ isomers compared to their most stable $\mathrm{Ama}^{+}(\mathrm{I}-\mathrm{III})-\mathrm{Ar}$ isomers calculated at the B3LYP-D3/cc-pVTZ level.

Figure S3. IRPD spectrum of $\mathrm{Ama}^{+} \mathrm{Ar}$ compared to linear IR absorption spectra of $\mathrm{Ama}^{+}(\mathrm{I})-\mathrm{Ar}$ isomers (I-VIII) calculated at B3LYP-D3/cc-pVTZ level. Relative zero-point corrected energies $E_{0}$ are given in $\mathrm{kJmol}^{-1}$.

Figure S4. IRPD spectrum of $\mathrm{Ama}^{+} \mathrm{Ar}$ compared to linear IR absorption spectra of $\mathrm{Ama}^{+}(\mathrm{II})-\mathrm{Ar}$ isomers (I-VII) calculated at the B3LYP-D3/cc-pVTZ level. Relative zero-point corrected energies $E_{0}$ are given in $\mathrm{kJmol}^{-1}$.

Figure S5. IRPD spectrum of $\mathrm{Ama}^{+} \mathrm{Ar}$ compared to linear IR absorption spectra of $\mathrm{Ama}^{+}(\mathrm{III})-\mathrm{Ar}$ isomers (I-VII) calculated at the B3LYP-D3/cc-pVTZ level. Relative zero-point corrected energies $E_{0}$ are given in $\mathrm{kJmol}^{-1}$.

Figure S6. HOMO orbitals of Ama and $\mathrm{Ama}^{+}(\mathrm{I}-\mathrm{III})$ isomers evaluated at the B3LYP-D3/cc-pVTZ level.

Figure S7. NBO charge distribution (in me) of $\mathrm{Ama}$ and $\mathrm{Ama}^{+}(\mathrm{I}-\mathrm{III})$ isomers in their ground electronic states calculated at the B3LYP-D3/cc-pVTZ level.

Figure S8. IR absorption spectrum of Ama compared to IR absorption spectra of $\mathrm{Ama}^{+}(\mathrm{I}-\mathrm{III})$ isomers calculated at B3LYP-D3/cc-pVTZ level.

Figure S9. NBO spin density distribution of $\mathrm{Ama}$ and $\mathrm{Ama}^{+}(\mathrm{I}-\mathrm{III})$ isomers in their ground electronic states calculated at the B3LYP-D3/cc-pVTZ level.

Figure S10. Equilibrium structures (in $\AA$ and degrees) of $A d a$ and $\mathrm{Ada}^{+}(\mathrm{I}-\mathrm{III})$ calculated at the B3LYP-D3/ccpVTZ level.

Figure S11. Potential energy surface of $\mathrm{Ama}^{+}$isomers (I-III) and $\mathrm{Ada}^{+}$isomers (I-III) for the rearrangement process upon ionization.

Figure S12 NBO charge distribution (in me) of $\mathrm{Ada}$ and $\mathrm{Ada}^{+}(\mathrm{I}-\mathrm{III})$ isomers in their ground electronic states calculated at the B3LYP-D3/cc-pVTZ level.

Figure S13. NBO spin density distribution of $\mathrm{Ada}$ and $\mathrm{Ada}^{+}(\mathrm{I}-\mathrm{III})$ isomers in their ground electronic states calculated at the B3LYP-D3/cc-pVTZ level.

Figure S14. HOMO orbitals of Ada and $\mathrm{Ada}^{+}(\mathrm{I}-\mathrm{III})$ isomers evaluated at the B3LYP-D3/cc-pVTZ level.

Cartesian coordinates and energies of all relevant structures 


\section{Experimental and Theoretical Methods}

IRPD spectra of $\mathrm{Ama}^{+} \mathrm{Ar}$ are recorded in a quadrupole tandem mass spectrometer (QMS1/2) coupled to an electron ionization cluster source. ${ }^{1,2}$ Ama $^{+} \mathrm{Ar}$ clusters are generated in a pulsed supersonic plasma expansion by electron and/or chemical ionization of a heated Ama sample (Sigma Aldrich, $>97 \%, \mathrm{~T} \sim 130^{\circ}$ ) seeded in Ar carrier gas at 10-20 bar stagnation pressure close to the nozzle orifice and subsequent threebody clustering reactions. The filaments of the electron impact source are operated with an offset voltage of up to $200 \mathrm{~V}$, which provides the upper limit for the kinetic energy of the electrons, $E_{k i n}(e)$. The $\operatorname{Ama}^{+} A r$ clusters are mass selected by QMS1 and irradiated with a tuneable IR laser pulse $\left(v_{\mathbb{R}}\right)$ in an octupole ion trap generated by a pulsed OPO laser with a bandwidth of $1 \mathrm{~cm}^{-1}$. Resonant vibrational excitation of the cluster leads to the loss of the Ar ligand. The produced $\mathrm{Ama}^{+}$fragments are selected by QMS2 and monitored by a Daly detector as a function of the laser frequency to obtain IRPD spectra of Ama ${ }^{+} A r$. To account for background signal arising from metastable decay, the ion source is triggered at twice the laser frequency $(10 \mathrm{~Hz})$ and signals from alternating triggers are subtracted.

Geometries, energies, and harmonic IR spectra of stable $\mathrm{Ama}^{+}$isomers and their corresponding clusters are calculated at the unrestricted B3LYP-D3/cc-pVTZ level. ${ }^{3}$ Relative energies and binding energies $\left(E_{\mathrm{e}}, D_{\mathrm{e}}\right)$ are corrected for harmonic zero-point vibrational energy to yield $E_{0}$ and $D_{0}$. Harmonic frequencies of $\mathrm{NH} / \mathrm{OH}$ modes are scaled by a factor of 0.9491 derived from fitting $\mathrm{NH}$ stretch frequencies of neutral Ama to experimental values. ${ }^{4}$ Since the $\mathrm{CH}$ cage structure of Ama and Ada are similar, the $\mathrm{CH}$ stretch frequencies are scaled by a factor of 0.9618 , derived from the experimental data of $\mathrm{Ada}^{+} \mathrm{He}_{2}{ }^{5}$ The $\mathrm{CH}_{2} / \mathrm{NH}_{2}$ bending modes in the fingerprint range below $2000 \mathrm{~cm}^{-1}$ are scaled by 0.9732 derived from fitting the $\mathrm{NH}_{2}$ bending frequency of the related aniline cation to the experimental value of the $\mathrm{NH}_{2}$ bending overtone. ${ }^{6}$ The use of three scaling factors accounts for the somewhat different anharmonicities of the various stretching and bending modes. Natural bond orbital (NBO) analysis is used to evaluate the charge distribution, charge transfer, and spin density. ${ }^{7,8}$

\section{References}

(1) Dopfer, O. Z. Phys. Chem. 2005, 219, 125-168.

(2) Dopfer, O. Int. Rev. Phys.I Chem. 2003, 22, 437-495.

(3) Frisch, M. et al. Gaussian16, Revision C.01; Gaussian, Inc.: Wallinford, CT, 2016.

(4) Linstrom, P. J.; Mallard, W. G. NIST Chemistry WebBook, NIST Standards and Technology, Gaithersburg, MD, http://webbook.nist.gov/ (accessed 2021-10-1).

(5) Patzer, A.; Schütz, M.; Möller, T.; Dopfer, O. Angew. Chem. Int. Ed. 2012, 51, 4925-4929.

(6) Solcà, N.; Dopfer, O. Eur. Phys. J. D 2002, 20, 469-480.

(7) Glendening, E. D.; Badenhoop, J. K.; Reed, A. E.; Carpenter, J. E.; Bohmann, J. A.; Morales, C. M.; Landis, C. R.; Weinhold, F., NBO, version 6.0, Theoretical Chemistry Institute, University of Wisconsin: Madison, WI, 2013.

(8) Reed, A. E.; Curtiss, L. A.; Weinhold, F. Chem. Rev. 1988, 88, 899-926. 
Table S1. Computed vibrational frequencies (in $\mathrm{cm}^{-1}, \mathrm{~B} 3 \mathrm{LYP}-\mathrm{D} 3 / \mathrm{cc}-\mathrm{pVTZ}$ ) of $\mathrm{Ama}^{+}(\mathrm{I}-\mathrm{III})$ compared to experimental values of $\mathrm{Ama}^{+} \mathrm{Ar}$ (Figure 1).

\begin{tabular}{|c|c|c|c|c|}
\hline Mode $^{[a]}$ & $\operatorname{Ama}^{+}(I)^{[\mathrm{b}]}$ & $\mathrm{Ama}^{+}(\mathrm{II})^{[\mathrm{b}]}$ & $\mathrm{Ama}^{+}(\mathrm{III})^{[\mathrm{b]}}$ & $\begin{array}{c}\mathrm{Ama}^{+}-\mathrm{Ar} \\
\text { Exp. }^{[\mathrm{c}]}\end{array}$ \\
\hline$\tau_{\mathrm{CH} 2}, \gamma_{\mathrm{CH} 2}$ & & & $\begin{array}{c}1226 \\
1218(2) \\
1227(10)\end{array}$ & $R 1228(15)$ \\
\hline$\rho_{\mathrm{NH} 2}, \tau_{\mathrm{CH} 2}, \gamma_{\mathrm{CH} 2}$ & $\begin{array}{c}1226 \\
1226(22) \\
1243(0.001)\end{array}$ & & & $R 1228$ (15) \\
\hline$\gamma_{\mathrm{CH} 2}$ & & $1247(22)$ & & $R 1228(15)$ \\
\hline$\tau_{\mathrm{CH} 2}, \gamma_{\mathrm{CH} 2}$ & $\begin{array}{c}\mathbf{1 2 7 4} \\
1254(1.3) \\
1271(0.01) \\
1273(0.1) \\
1273(26) \\
1296(5) \\
1307(0.4) \\
\end{array}$ & $\begin{array}{c}1285 \\
1283(1) \\
1285(4)\end{array}$ & $\begin{array}{c}1301 \\
1260(1) \\
1280(3) \\
1292(1) \\
1301(14) \\
1316(5)\end{array}$ & \\
\hline$\gamma_{\mathrm{CH} 2}$ & $\begin{array}{c}1346 \\
1319(0.1) \\
1325(2) \\
1345(2) \\
1345(1) \\
1352(2) \\
1362(0.2) \\
\end{array}$ & $\begin{array}{c}1310 \\
1306(3) \\
1311(2) \\
1323(1) \\
1330(0.1)\end{array}$ & $\begin{array}{c}1336 \\
1332(4) \\
1338(7) \\
1348(2)\end{array}$ & S $1368(35)$ \\
\hline$\rho_{\mathrm{NH} 2}, \gamma_{\mathrm{CH} 2}, \gamma_{\mathrm{CH} 3}$ & & & $\begin{array}{c}1372 \\
1363(15) \\
1378(12) \\
1380(4) \\
\end{array}$ & S $1368(35)$ \\
\hline$\rho_{\mathrm{NH} 2}$ & & $1397(10)$ & & S $1368(35)$ \\
\hline$\beta_{\mathrm{CH} 2}, \beta_{\mathrm{CH} 3}$ & $\begin{array}{c}1458 \\
1435(5) \\
1439(1) \\
1450(15) \\
1460(12) \\
1463(14) \\
1484(1)\end{array}$ & $\begin{array}{c}1441 \\
1430(1) \\
1438(17) \\
1440(20) \\
1469 \\
1464(3) \\
1468(17) \\
1473(3)\end{array}$ & $\begin{array}{c}1440 \\
1421(6) \\
1430(0.1) \\
1430(16) \\
1441(11) \\
1442(30) \\
1447(5) \\
1467(5)\end{array}$ & $P 1458(25)$ \\
\hline$\left(\beta_{\mathrm{NH} 2} / v_{\mathrm{CN}}\right)^{\mathrm{s}}$ & & $1542(4)$ & $1500(93)$ & $T 1551(7)$ \\
\hline$\beta_{\mathrm{NH} 2}$ & $1595(70)$ & & & $Q_{1} 1599(6)$ \\
\hline$\left(\beta_{\mathrm{NH} 2} / v_{\mathrm{CN}}\right)^{\mathrm{a}}$ & & & $1641(216)$ & $\boldsymbol{Q}_{\mathbf{2}} 1664(13)$ \\
\hline$\left(\beta_{\mathrm{NH} 2} / v_{\mathrm{CN}}\right)^{\mathrm{a}}$ & & $1670(208)$ & & $Q_{3} 1717(7)$ \\
\hline$v_{\mathrm{CH}}$ & & $2821(8)$ & & A 2842 (4) \\
\hline$v_{\mathrm{CH}}$ & $2840(75)$ & & & B 2864 (11) \\
\hline$v_{\mathrm{CH} 2}, v_{\mathrm{CH} 3}$ & & & $\begin{array}{c}2878 \\
2858(0.3) \\
2873(2) \\
2878(16) \\
\end{array}$ & B 2864 (11) \\
\hline$v_{\mathrm{CH} 2}, v_{\mathrm{CH} 3}$ & $\begin{array}{c}2920 \\
2908(4) \\
2912(16) \\
2921(16) \\
2923(20) \\
2925(10)\end{array}$ & $\begin{array}{c}2901 \\
2898(10) \\
2901(40) \\
2926 \\
2916(0.5) \\
2921(8) \\
2929(11) \\
\end{array}$ & $\begin{array}{c}2898(0) \\
2905(9) \\
2926 \\
2925(10) \\
2930(6)\end{array}$ & C 2920 (6) \\
\hline$v_{\mathrm{CH}}, v_{\mathrm{CH} 2}$ & $\begin{array}{c}2955 \\
2949(5) \\
2950(28)\end{array}$ & & & D 2947 (25) \\
\hline
\end{tabular}




\begin{tabular}{|c|c|c|c|c|}
\hline & $\begin{array}{c}2951(1) \\
2952(10) \\
2961(6) \\
2962(11) \\
2966(4) \\
2971(27)\end{array}$ & & & \\
\hline$v_{\mathrm{CH} 2}, v_{\mathrm{CH}}, v_{\mathrm{CH} 3}$ & $\begin{array}{c}2961(6) \\
2962(11)\end{array}$ & $2956(13)$ & $\begin{array}{c}2961 \\
2951(5) \\
2953(11) \\
2957(11) \\
2959(20) \\
2963(7) \\
2965(28) \\
2966(4) \\
2977(17) \\
\end{array}$ & E 2959 (6) \\
\hline$v_{\mathrm{CH} 2}$ & $2971(27)$ & $\begin{array}{c}2972 \\
2955(0) \\
2956(13) \\
2970(25) \\
2971(5) \\
2974(8) \\
2974(9) \\
2976(8)\end{array}$ & $2977(17)$ & F 2978 (4) \\
\hline$v_{\mathrm{CH} 2}$ & $3024(6)$ & $3021(6)$ & & G 3008 (6) \\
\hline$v_{\mathrm{CH} 2}$ & & $3121(4)$ & & H $3151(9)$ \\
\hline $2 \beta_{\mathrm{NH} 2}$ & 3190 & & & H3151 (9) \\
\hline$\left(\beta_{\mathrm{NH} 2} / v_{\mathrm{CN}}\right)^{\mathrm{a}+\mathrm{s}}$ & & & 3140 & H3151 (9) \\
\hline $2\left(\beta_{\mathrm{NH} 2} / v_{\mathrm{CN}}\right)^{\mathrm{a}}$ & & & 3282 & I3267 (8) \\
\hline$\left(\beta_{\mathrm{NH} 2} / v_{\mathrm{CN}}\right)^{\mathrm{a}+\mathrm{s}}$ & & 3212 & & I $3267(8)$ \\
\hline$v_{\mathrm{NH} 2}^{\mathrm{s}}$ & $3322(223)$ & & & $J_{1} 3321(16)$ \\
\hline$v_{\mathrm{NH} 2}{ }^{\mathrm{s}}$ & & 3329 (131) & & $J_{2} 3345(8)$ \\
\hline$v_{\mathrm{NH} 2}{ }^{\mathrm{s}}$ & & & 3358 (173) & $J_{3} 3381(17)$ \\
\hline$v_{\mathrm{NH} 2}{ }^{a}$ & $3438(68)$ & & & $K_{1} 3425(9)$ \\
\hline$v_{\mathrm{NH} 2}{ }^{a}$ & & $3433(76)$ & & $K_{2} 3451(7)$ \\
\hline$v_{\mathrm{NH} 2}{ }^{a}$ & & & 3467 (63) & $K_{3} 3477(11)$ \\
\hline
\end{tabular}

[a] Stretching $(v)$, bending $(\beta)$, torsion $(\tau)$, wagging $(\gamma)$, rocking $(\rho)$.

${ }^{[b]}$ IR intensities in $\mathrm{kmmol}^{-1}$ are given in parentheses. Harmonic wavenumbers are scaled by 0.9732 $(\beta, \tau, \gamma, \rho), 0.9618\left(v_{\mathrm{CHn}}\right)$ and $0.9491\left(v_{\mathrm{NH} 2}\right)$. Positions of convoluted bands are given in bold.

[c] The experimental values are given with peak width (fwhm, in parenthesis) and assigned to the most dominant vibrations. 
Table S2. Various energies (in $\mathrm{kJmol}^{-1}$ ) of $\mathrm{Ama}^{+}(\mathrm{I}-\mathrm{III}), \mathrm{Ama}^{+}(\mathrm{I}-\mathrm{III})-\mathrm{Ar}$ and $\mathrm{Ada}^{+}(\mathrm{I}-\mathrm{III})$ isomers calculated at the B3LYP-D3/cc-pVTZ level.

\begin{tabular}{|c|c|c|c|c|}
\hline & $E_{0}$ & $E_{\mathrm{e}}$ & $G$ & $D_{0}$ \\
\hline $\operatorname{Ama}^{+}(\mathrm{I})$ & 45.88 & 40.59 & 51.86 & \\
\hline $\mathrm{Ama}^{+}(\mathrm{II})$ & 87.38 & 88.04 & 85.92 & \\
\hline $\mathrm{Ama}^{+}(\mathrm{III})$ & 0 & 0 & 0 & \\
\hline Ama $^{+} T S(I-I I)$ & 99.95 & 100.13 & 101.38 & \\
\hline Ama $^{+} T S(I I-I I I)$ & 169.01 & 178.67 & 172.22 & \\
\hline $\operatorname{Ama}^{+}(\mathbf{I})-\operatorname{Ar}(\mathbf{I})$ & 46.30 & 40.54 & 50.43 & 8.36 \\
\hline $\mathrm{Ama}^{+}(\mathrm{II})-\operatorname{Ar}(\mathrm{I})$ & 87.27 & 87.92 & 84.28 & 8.90 \\
\hline $\mathrm{Ama}^{+}(\mathrm{III})-\operatorname{Ar}(\mathrm{I})$ & 0 & 0 & 0 & 8.79 \\
\hline $\mathrm{Ama}^{+} \mathrm{TS}(\mathrm{I}-\mathrm{II})-\mathrm{Ar}$ & 99.16 & 99.95 & 97.95 & \\
\hline Ama $^{+} T S(I I-I I I)-A r$ & 169.14 & 179.85 & 179.85 & \\
\hline $\operatorname{Ada}^{+}(\mathbf{I})$ & 5.37 & 10.96 & 12.43 & \\
\hline $\mathrm{Ada}^{+}(\mathrm{II})$ & 152.95 & 162.74 & 148.41 & \\
\hline $\operatorname{Ada}^{+}(\mathrm{III})$ & 0 & 0 & 0 & \\
\hline $\mathrm{Ada}^{+} \mathrm{TS}(\mathrm{I}-\mathrm{II})$ & 162.03 & 170.02 & 158.46 & \\
\hline $\mathrm{Ada}^{+} \mathrm{TS}(\mathrm{II}-\mathrm{III})$ & 190.77 & 202.91 & 188.63 & \\
\hline
\end{tabular}


Table S3. Various energies (in $\mathrm{kJmol}^{-1}$ ) of $\mathrm{Ama}^{+}(\mathrm{I}-\mathrm{III})-\mathrm{Ar}(\mathrm{I}-\mathrm{VIII})$ isomers calculated at the B3LYP-D3/cc-pVTZ level. $E_{0}{ }^{\text {total }}$ denotes the energy difference to the global minimum $\mathrm{Ama}^{+}(\mathrm{III})$.

\begin{tabular}{|c|c|c|c|c|c|}
\hline & $E_{0}$ & $E_{\mathrm{e}}$ & $G$ & $D_{0}$ & $E_{0}^{\text {total }}$ \\
\hline $\operatorname{Ama}^{+}(\mathrm{I})-\operatorname{Ar}(\mathrm{I})$ & 0 & 0 & 0 & 8.36 & 46.31 \\
\hline $\operatorname{Ama}^{+}(\mathbf{I})-\operatorname{Ar}(\mathrm{II})$ & 0.67 & 0.75 & -0.56 & 7.69 & 46.98 \\
\hline $\operatorname{Ama}^{+}(\mathrm{I})-\operatorname{Ar}(\mathrm{III})$ & 1.17 & 1.06 & 2.70 & 7.19 & 47.47 \\
\hline $\operatorname{Ama}^{+}(\mathbf{I})-\operatorname{Ar}(\mathbf{I V})$ & 2.08 & 2.16 & 2.00 & 6.28 & 48.39 \\
\hline $\mathrm{Ama}^{+}(\mathbf{I})-\operatorname{Ar}(\mathbf{V})$ & 2.21 & 3.31 & -1.80 & 6.15 & 48.51 \\
\hline $\operatorname{Ama}^{+}(\mathbf{I})-\operatorname{Ar}(\mathbf{V I})$ & 2.72 & 3.23 & 2.32 & 5.65 & 49.02 \\
\hline $\operatorname{Ama}^{+}(\mathrm{I})-\operatorname{Ar}(\mathbf{V I I})$ & 2.85 & 3.61 & 1.90 & 5.51 & 49.16 \\
\hline $\operatorname{Ama}^{+}(\mathbf{I})-\operatorname{Ar}(\mathbf{V I I I})$ & 2.91 & 3.69 & -0.13 & 5.45 & 49.22 \\
\hline $\operatorname{Ama}^{+}(\mathbf{I I})-\operatorname{Ar}(\mathbf{I})$ & 0 & 0 & 0 & 8.90 & 87.27 \\
\hline $\operatorname{Ama}^{+}(\mathrm{II})-\operatorname{Ar}(\mathrm{II})$ & 0.94 & 1.38 & -0.48 & 7.97 & 88.21 \\
\hline $\operatorname{Ama}^{+}(\mathrm{II})-\operatorname{Ar}(\mathrm{III})$ & 1.81 & 2.32 & 0.71 & 7.08 & 89.09 \\
\hline $\operatorname{Ama}^{+}(\mathrm{II})-\operatorname{Ar}(\mathbf{I V})$ & 1.91 & 2.29 & 1.73 & 6.99 & 89.19 \\
\hline $\operatorname{Ama}^{+}(\mathrm{II})-\operatorname{Ar}(\mathbf{V})$ & 2.08 & 2.29 & 1.87 & 6.82 & 89.35 \\
\hline $\operatorname{Ama}^{+}(\mathrm{II})-\operatorname{Ar}(\mathbf{V I})$ & 2.26 & 2.10 & 2.97 & 6.64 & 89.53 \\
\hline $\operatorname{Ama}^{+}(\mathrm{II})-\operatorname{Ar}(\mathbf{V I I})$ & 4.84 & 5.20 & 3.78 & 4.06 & 92.11 \\
\hline $\mathrm{Ama}^{+}(\mathrm{III})-\operatorname{Ar}(\mathrm{I})$ & 0 & 0 & 0 & 8.79 & 0 \\
\hline $\mathrm{Ama}^{+}(\mathrm{III})-\operatorname{Ar}(\mathrm{II})$ & 1.57 & 2.44 & -3.27 & 7.22 & 1.57 \\
\hline $\mathrm{Ama}^{+}(\mathrm{III})-\operatorname{Ar}(\mathrm{III})$ & 1.90 & 2.49 & 0.01 & 6.89 & 1.90 \\
\hline $\mathrm{Ama}^{+}(\mathrm{III})-\operatorname{Ar}(\mathbf{I V})$ & 2.20 & 2.16 & 0.74 & 6.59 & 2.20 \\
\hline $\operatorname{Ama}^{+}(\mathrm{III})-\operatorname{Ar}(\mathbf{V})$ & 2.47 & 2.79 & 0.52 & 6.33 & 2.47 \\
\hline $\mathrm{Ama}^{+}(\mathrm{III})-\operatorname{Ar}(\mathbf{V I})$ & 2.75 & 3.00 & 1.32 & 6.04 & 2.75 \\
\hline $\mathrm{Ama}^{+}(\mathrm{III})-\operatorname{Ar}(\mathrm{VII})$ & 2.92 & 2.88 & 1.46 & 5.88 & 2.92 \\
\hline
\end{tabular}



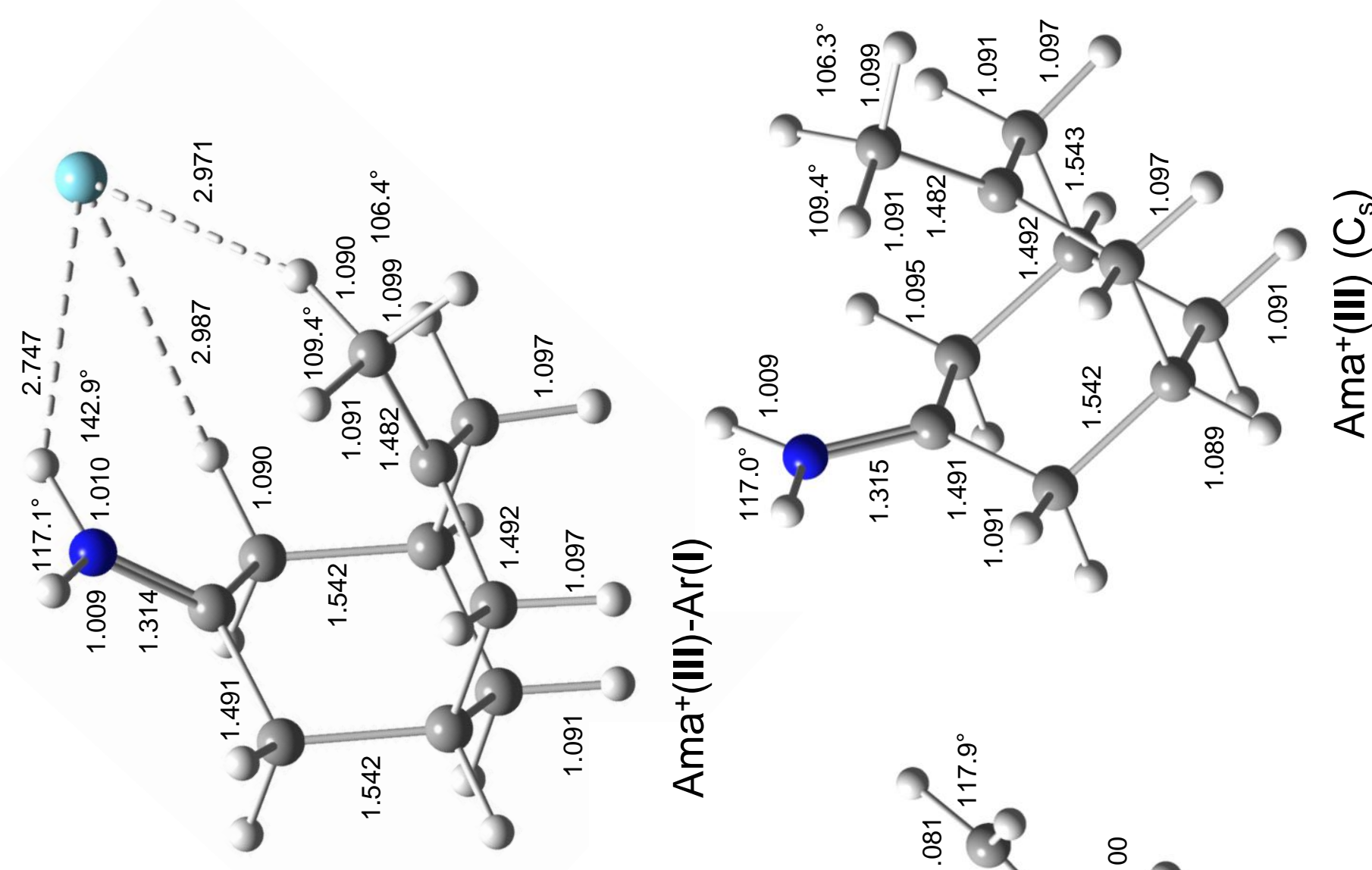

$\bar{c}$
$\frac{0}{\partial}$
$i \frac{0}{1}$
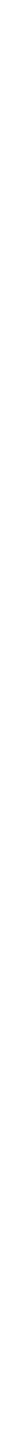

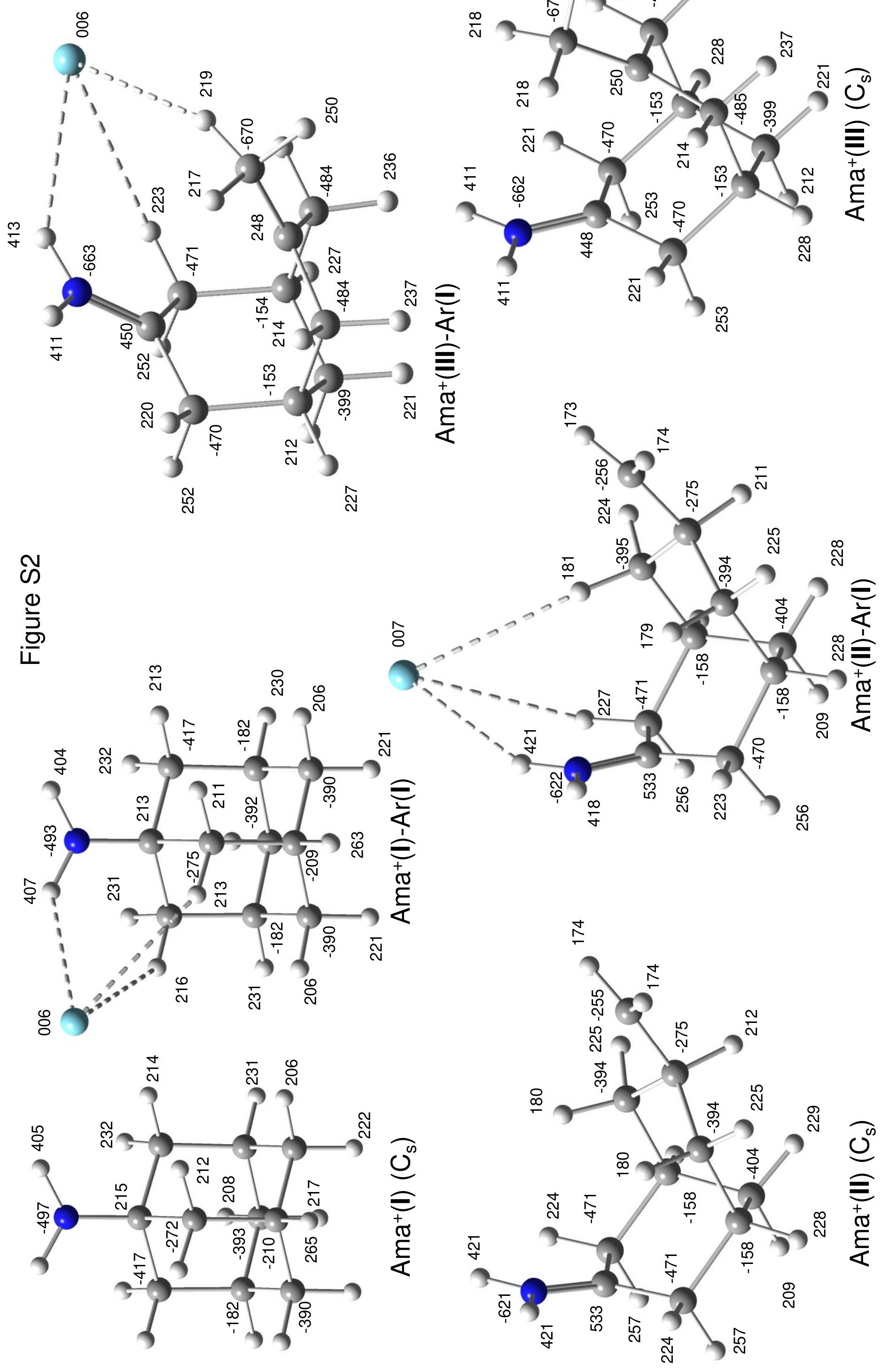
Figure S3

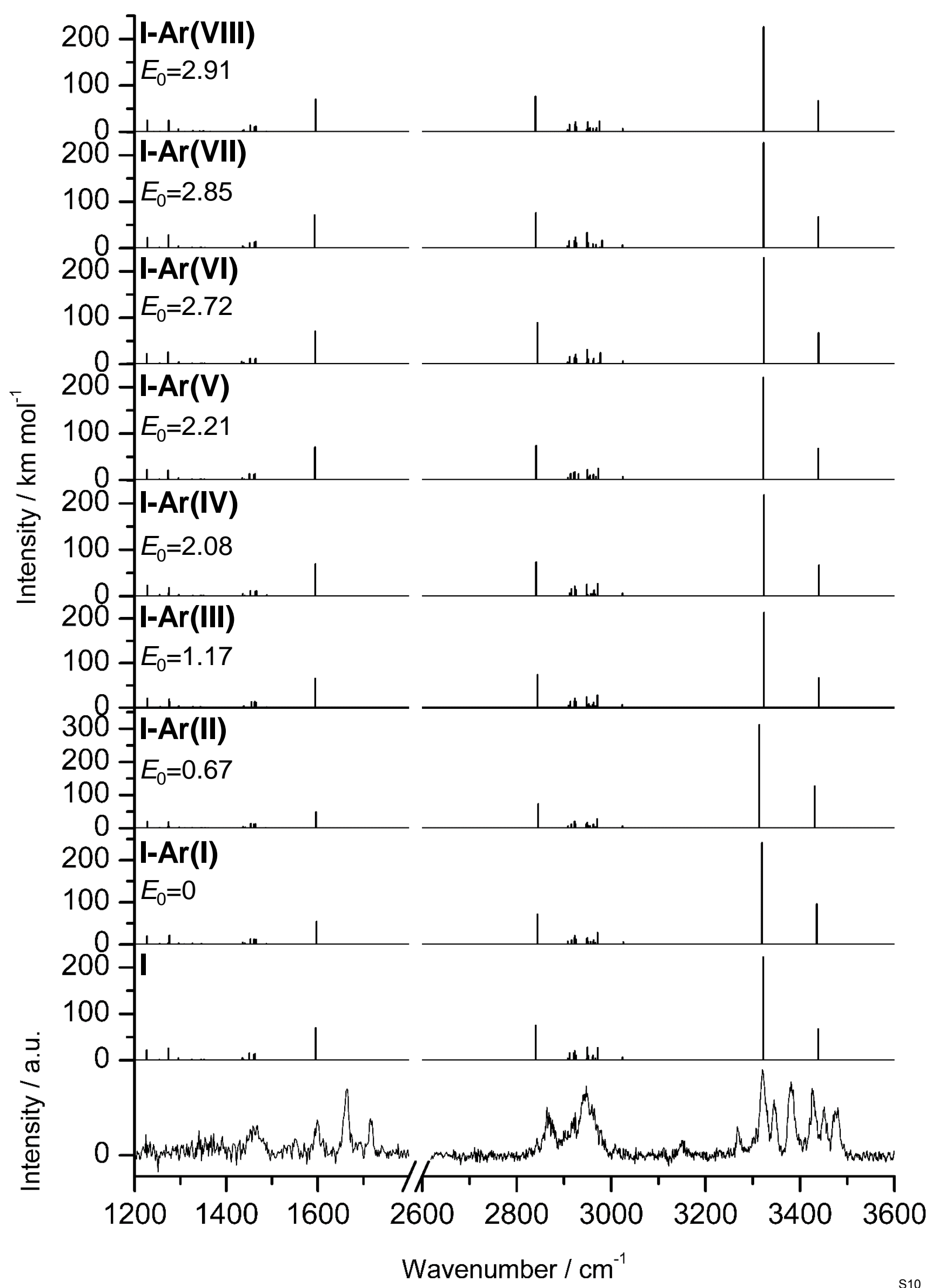


Figure S4

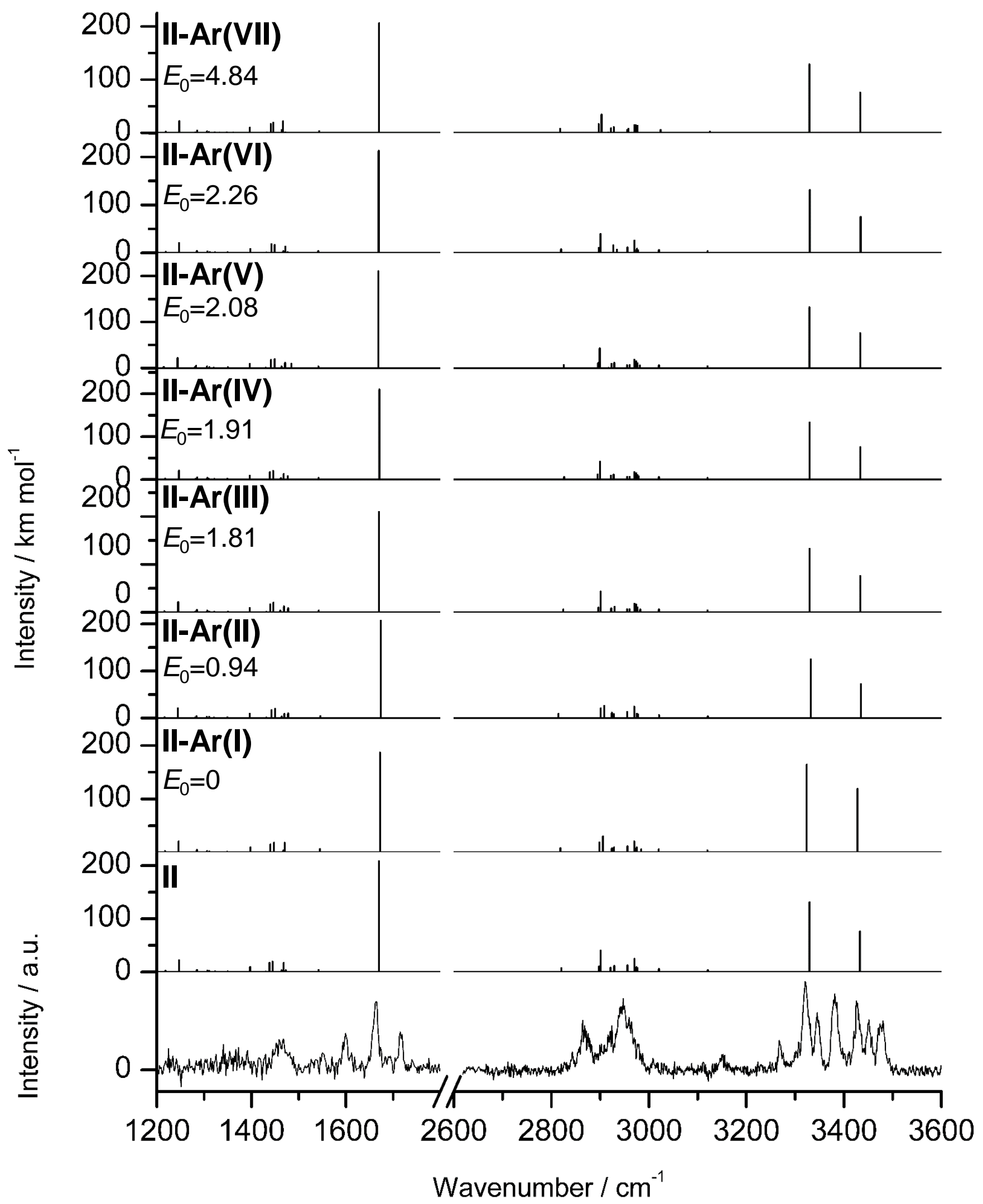


Figure S5

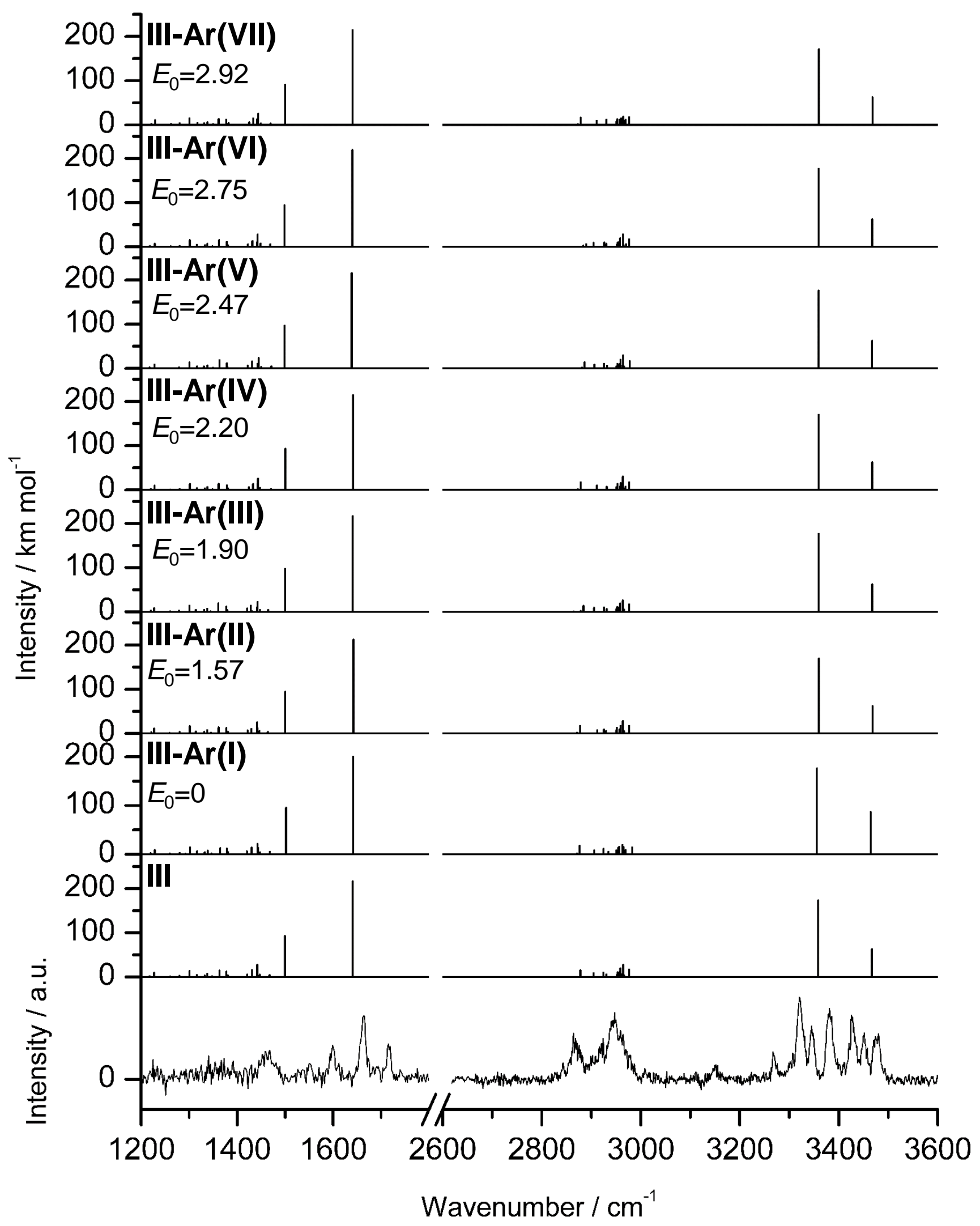




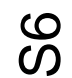

인
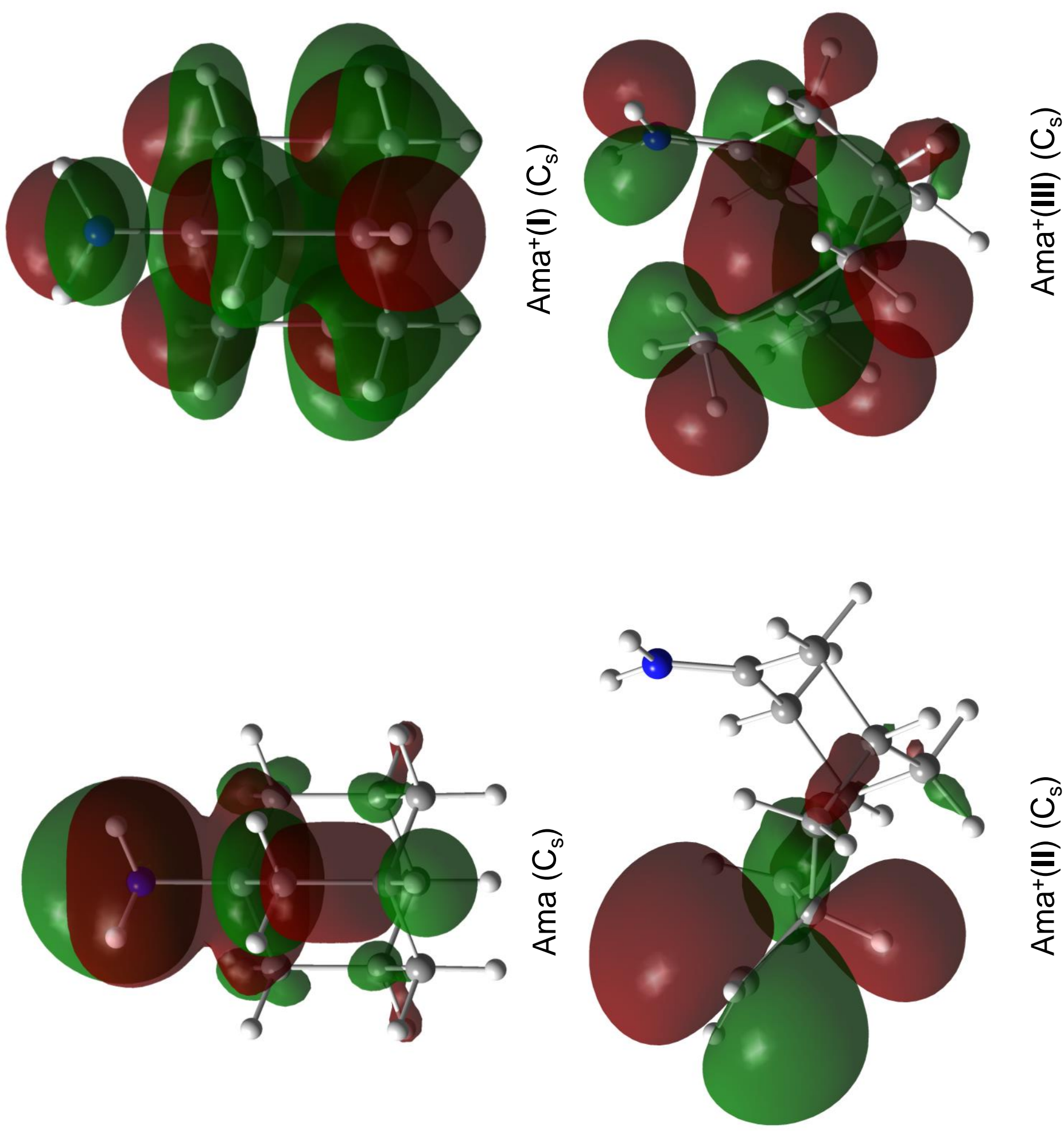

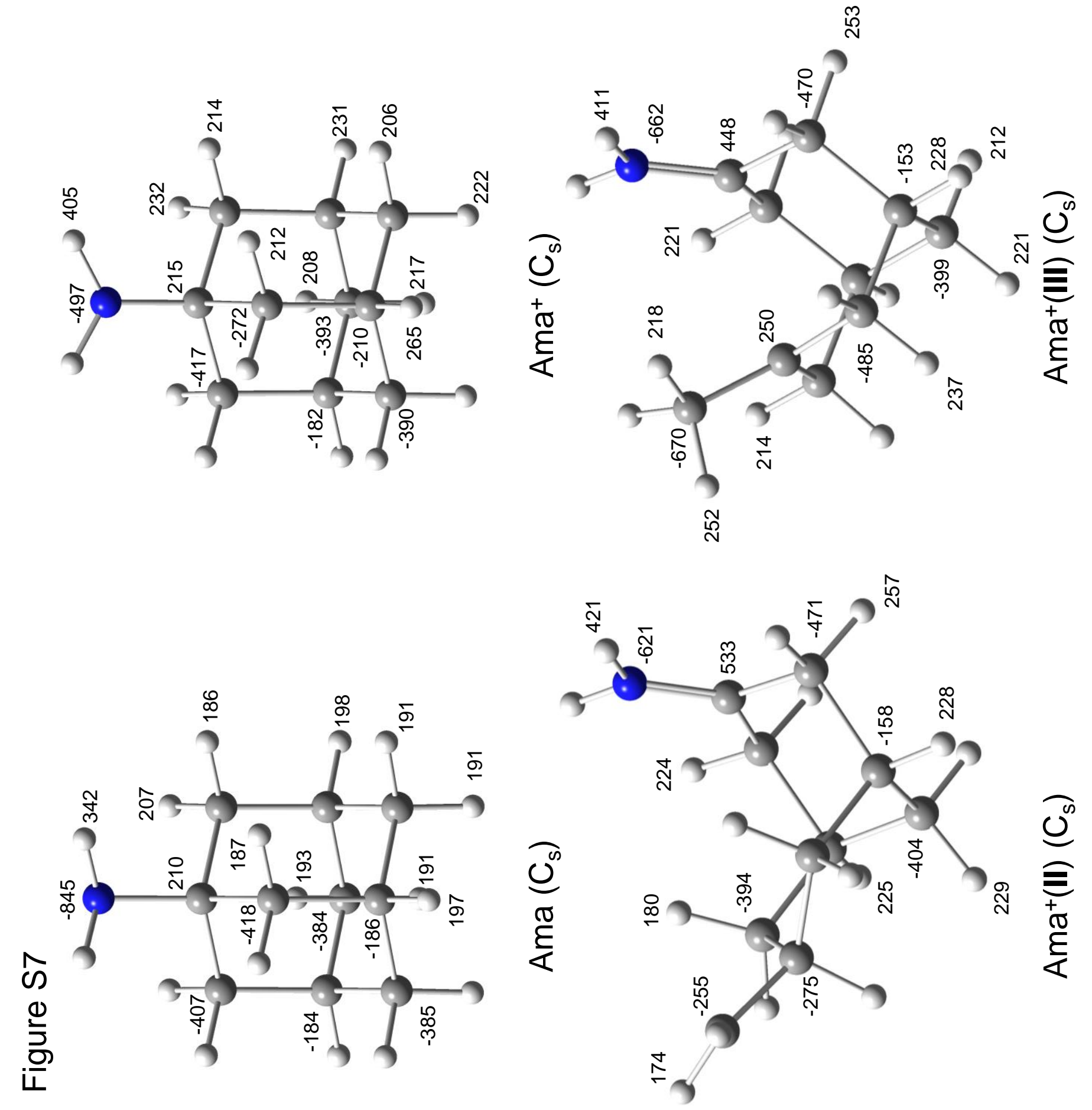
Figure S8

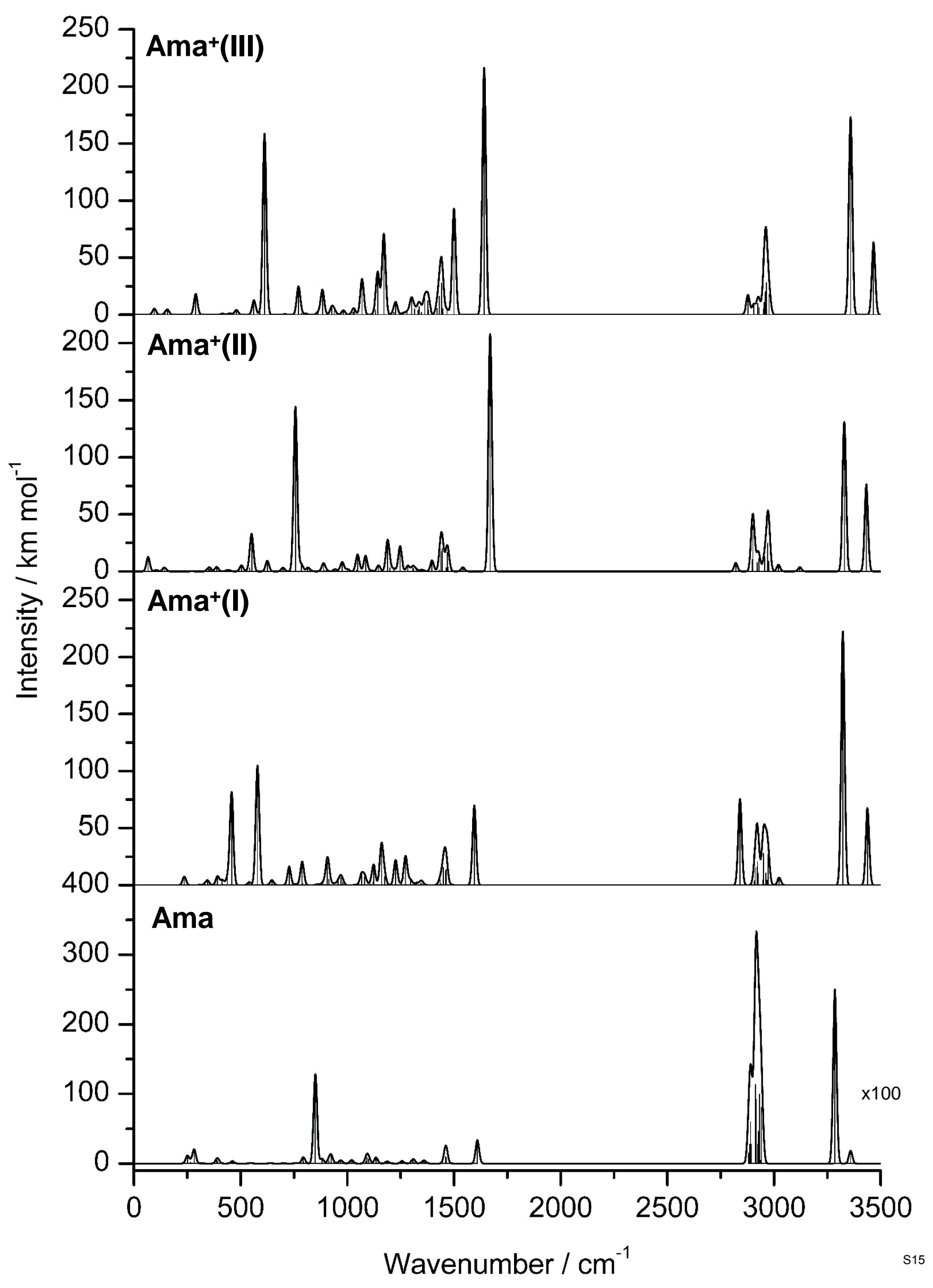




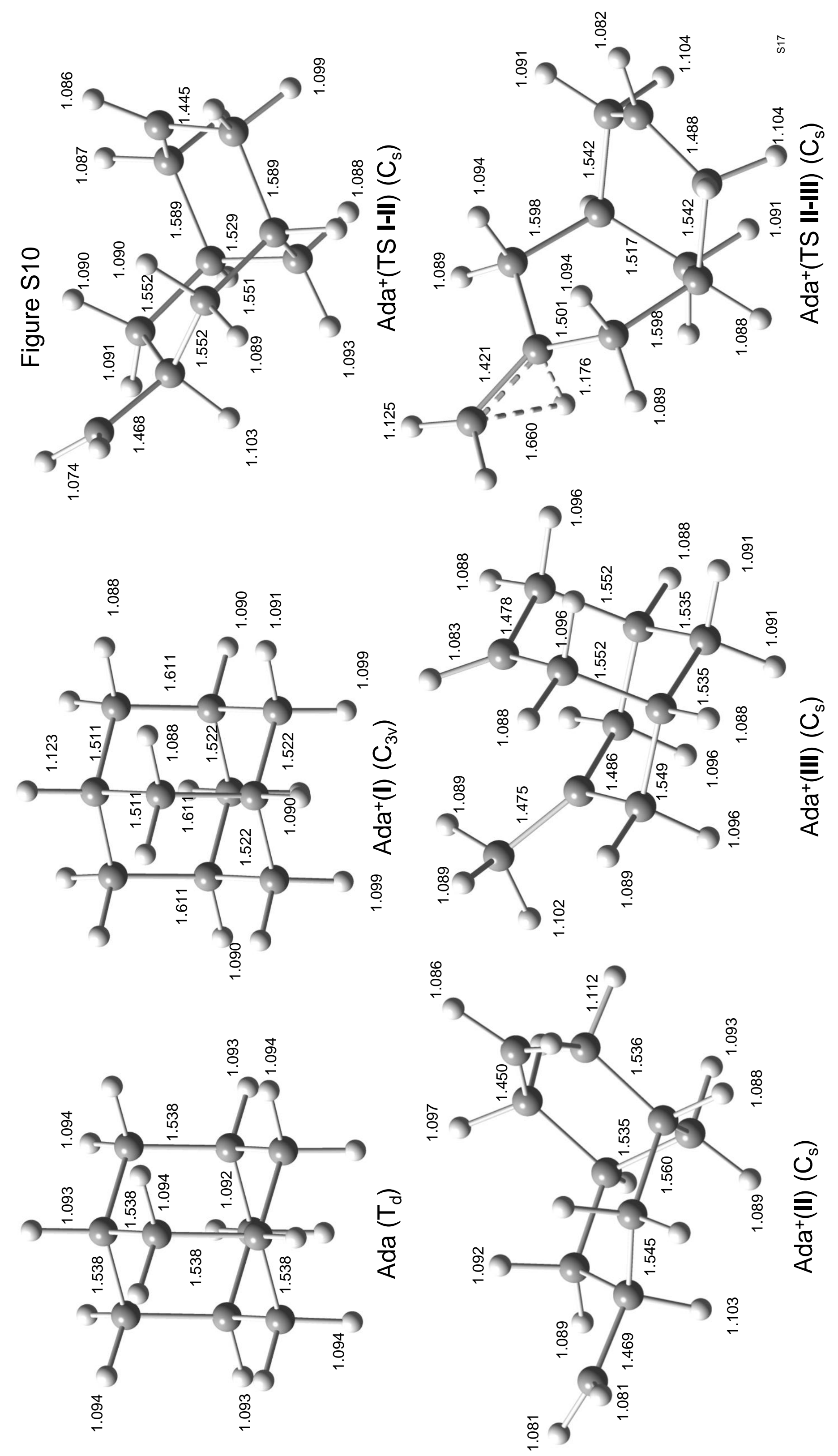


$\frac{5}{c}$
$\frac{0}{5}$
믄

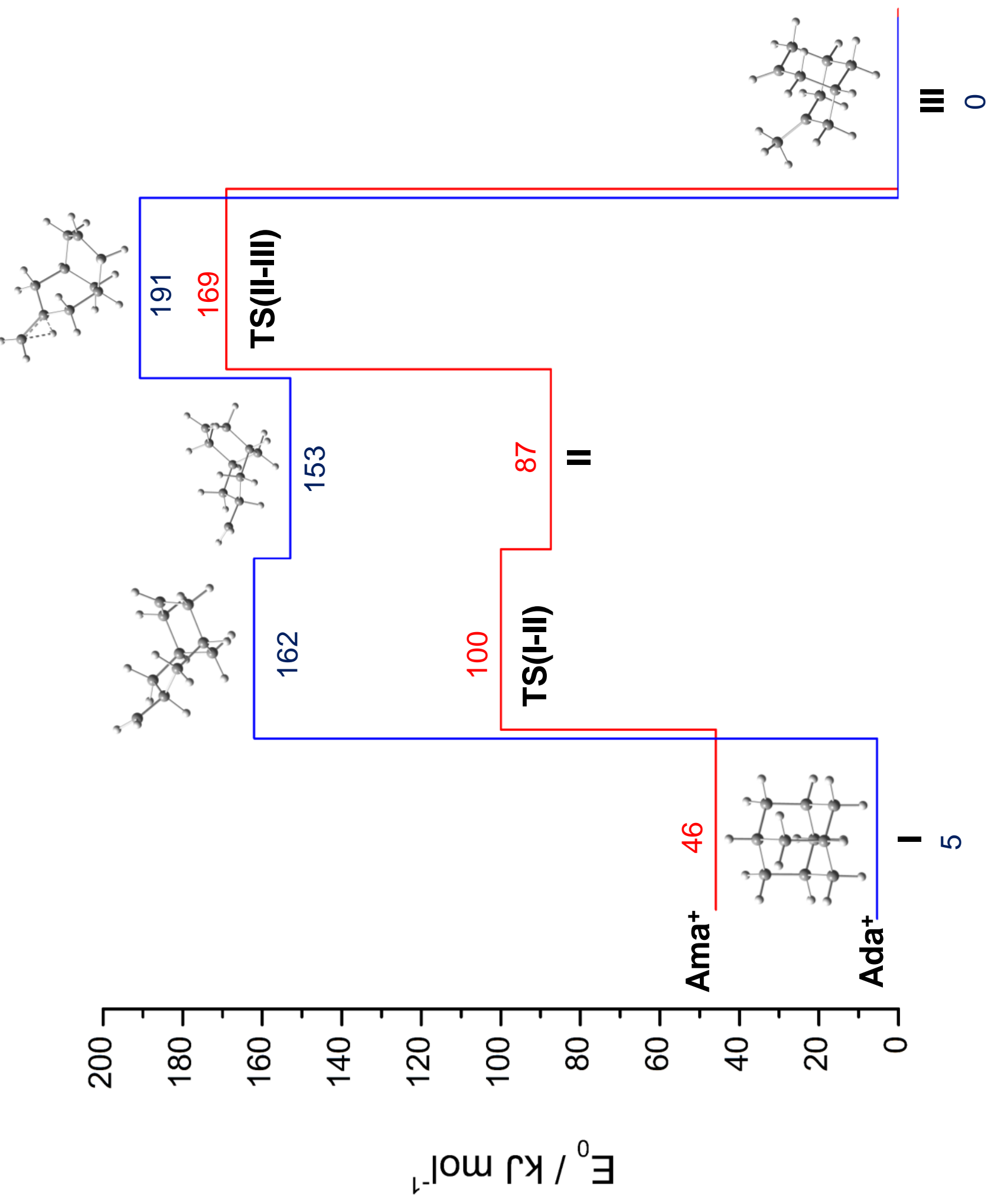




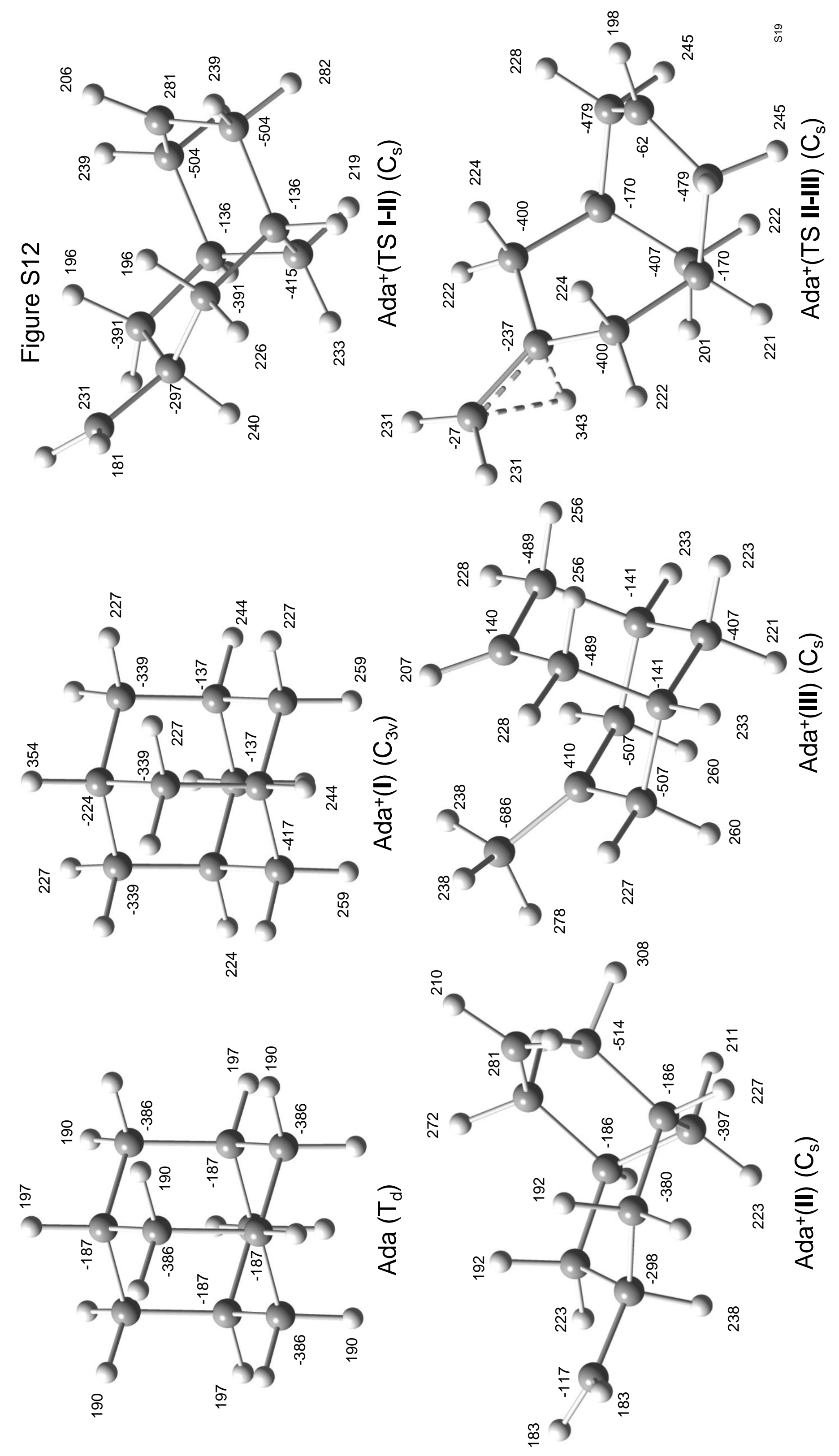


$\frac{n}{c}$
0
$\frac{0}{5}$
$\frac{0}{1}$
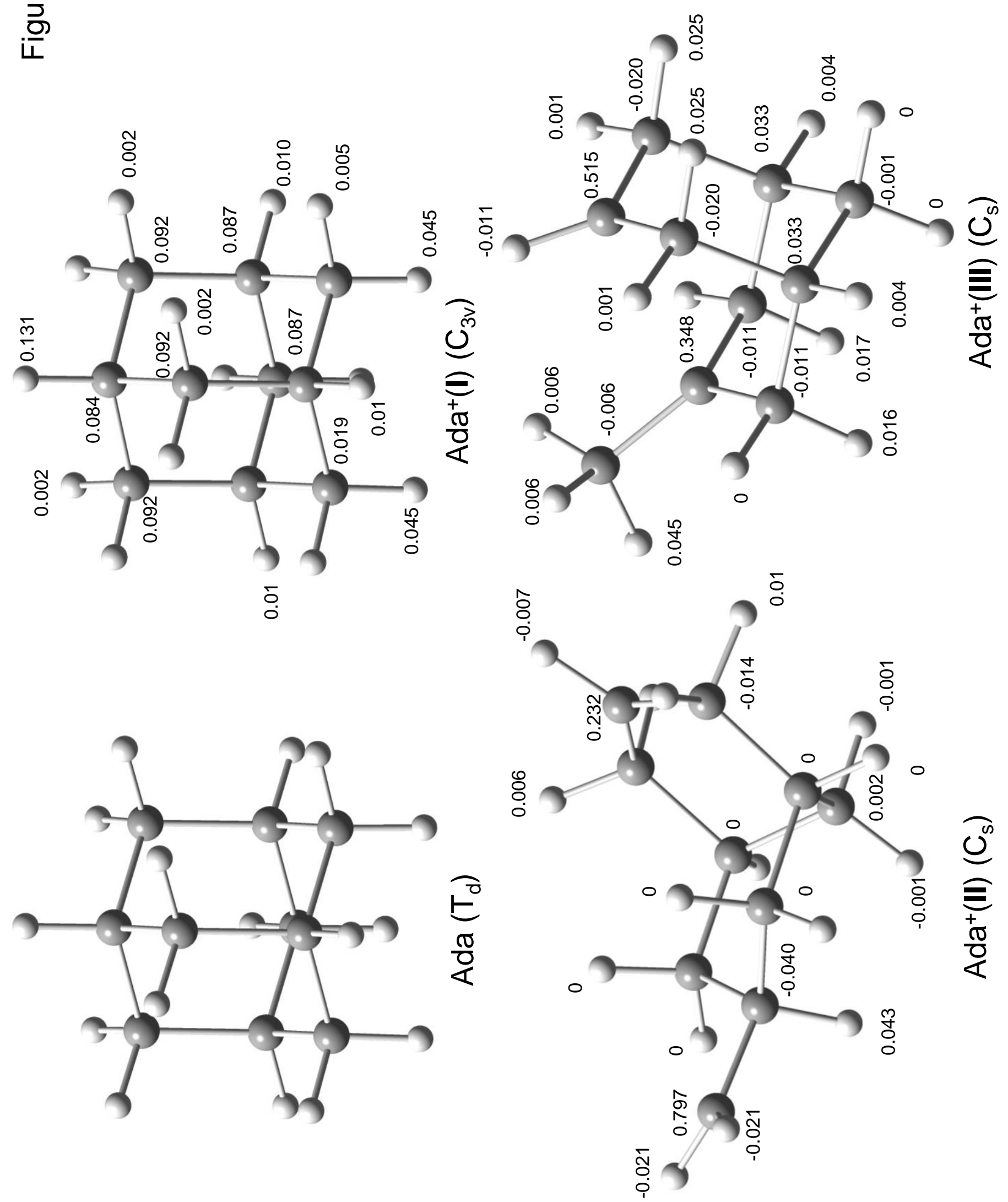
$\frac{\nabla}{c}$
$\frac{0}{5}$
$i \frac{0}{1}$
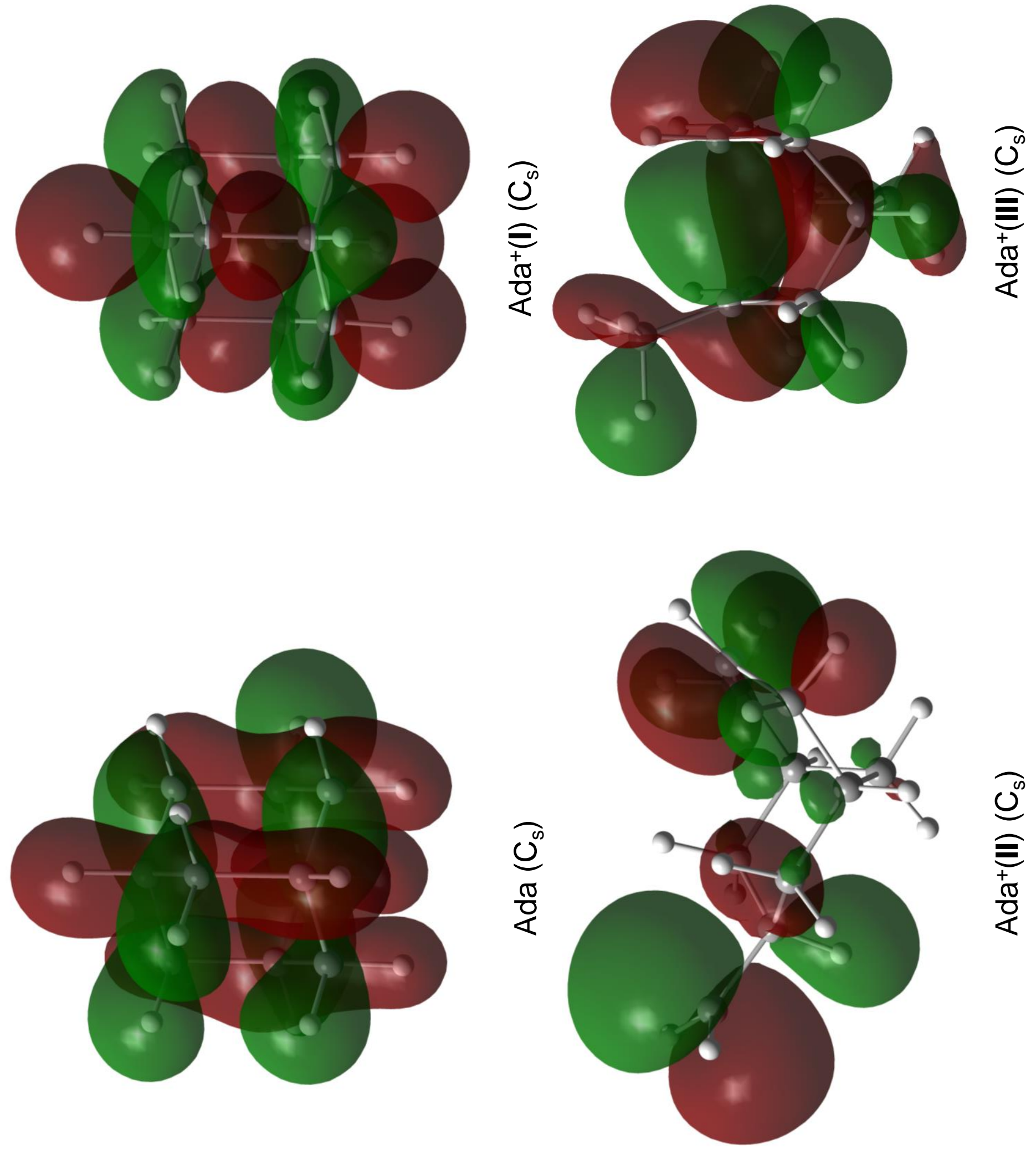
Ama

$\begin{array}{rrrrrr}1 & 6 & 0 & -1.011128 & 1.127081 & 1.255703 \\ 2 & 1 & 0 & -0.514261 & 1.502017 & 2.154656 \\ 3 & 1 & 0 & -2.038105 & 1.501352 & 1.276200 \\ 4 & 6 & 0 & -1.011128 & -0.410783 & 1.256075 \\ 5 & 1 & 0 & -1.521728 & -0.777686 & 2.149414 \\ 6 & 6 & 0 & -0.287239 & 1.641427 & 0.000000 \\ 7 & 1 & 0 & -0.281288 & 2.733996 & 0.000000 \\ 8 & 6 & 0 & 1.158945 & 1.116612 & 0.000000 \\ 9 & 1 & 0 & 1.696050 & 1.483603 & 0.880296 \\ 10 & 1 & 0 & 1.696050 & 1.483603 & -0.880296 \\ 11 & 6 & 0 & 0.437377 & -0.927013 & 1.249983 \\ 12 & 1 & 0 & 0.460778 & -2.018647 & 1.262920 \\ 13 & 1 & 0 & 0.962767 & -0.582715 & 2.146909 \\ 14 & 6 & 0 & 1.182128 & -0.427579 & 0.000000 \\ 15 & 6 & 0 & 0.437377 & -0.927013 & -1.249983 \\ 16 & 1 & 0 & 0.962767 & -0.582715 & -2.146909 \\ 17 & 1 & 0 & 0.460778 & -2.018647 & -1.262920 \\ 18 & 6 & 0 & -1.736812 & -0.921403 & 0.000000 \\ 19 & 1 & 0 & -2.774040 & -0.575535 & 0.000000 \\ 20 & 1 & 0 & -1.762177 & -2.014271 & 0.000000 \\ 21 & 6 & 0 & -1.011128 & 1.127081 & -1.255703 \\ 22 & 1 & 0 & -2.038105 & 1.501352 & -1.276200 \\ 23 & 1 & 0 & -0.514261 & 1.502017 & -2.154656 \\ 24 & 6 & 0 & -1.011128 & -0.410783 & -1.256075 \\ 25 & 1 & 0 & -1.521728 & -0.777686 & -2.149414 \\ 26 & 7 & 0 & 2.535235 & -0.993222 & 0.000000 \\ 27 & 1 & 0 & 3.048130 & -0.666622 & -0.812633 \\ 28 & 1 & 0 & 3.048130 & -0.666622 & 0.812633\end{array}$

Sum of electronic and zero-point Energies= Sum of electronic and thermal Energies= Sum of electronic and thermal Enthalpies= Sum of electronic and thermal Free Energies=
$-445.999992$

$-445.991746$

$-445.990802$

$-446.032017$

$\mathrm{Ama}^{+}(\mathrm{I})$

$\begin{array}{rrrrrr}1 & 6 & 0 & -1.009933 & 1.119927 & 1.260815 \\ 2 & 1 & 0 & -0.519111 & 1.502470 & 2.157785 \\ 3 & 1 & 0 & -2.034331 & 1.491912 & 1.270028 \\ 4 & 6 & 0 & -1.009933 & -0.418569 & 1.258258 \\ 5 & 1 & 0 & -1.512657 & -0.782974 & 2.154281 \\ 6 & 6 & 0 & -0.292961 & 1.610622 & 0.000000 \\ 7 & 1 & 0 & -0.245778 & 2.709641 & 0.000000 \\ 8 & 6 & 0 & 1.169160 & 1.189049 & 0.000000 \\ 9 & 1 & 0 & 1.708022 & 1.497285 & 0.892533 \\ 10 & 1 & 0 & 1.708022 & 1.497285 & -0.892533 \\ 11 & 6 & 0 & 0.436294 & -0.931980 & 1.280099 \\ 12 & 1 & 0 & 0.450942 & -2.023690 & 1.314481 \\ 13 & 1 & 0 & 0.966411 & -0.567549 & 2.163360 \\ 14 & 6 & 0 & 1.172297 & -0.507942 & 0.000000 \\ 15 & 6 & 0 & 0.436294 & -0.931980 & -1.280099 \\ 16 & 1 & 0 & 0.966411 & -0.567549 & -2.163360 \\ 17 & 1 & 0 & 0.450942 & -2.023690 & -1.314481 \\ 18 & 6 & 0 & -1.725250 & -0.938846 & 0.000000 \\ 19 & 1 & 0 & -2.762343 & -0.601654 & 0.000000 \\ 20 & 1 & 0 & -1.747492 & -2.030538 & 0.000000 \\ 21 & 6 & 0 & -1.009933 & 1.119927 & -1.260815 \\ 22 & 1 & 0 & -2.034331 & 1.491912 & -1.270028 \\ 23 & 1 & 0 & -0.519111 & 1.502470 & -2.157785 \\ 24 & 6 & 0 & -1.009933 & -0.418569 & -1.258258 \\ 25 & 1 & 0 & -1.512657 & -0.782974 & -2.154281 \\ 26 & 7 & 0 & 2.515855 & -0.841720 & 0.000000 \\ 27 & 1 & 0 & 3.044730 & -0.885075 & -0.862169 \\ 28 & 1 & 0 & 3.044730 & -0.885075 & 0.862169\end{array}$

Sum of electronic and zero-point Energies= Sum of electronic and thermal Energies=

Sum of electronic and thermal Enthalpies=

Sum of electronic and thermal Free Energies=
$-445.710234$

$-445.701526$

$-445.700582$

$-445.743151$ 
$\mathrm{Ama}^{+}(\mathrm{II})$

$\begin{array}{rrrrrr}1 & 6 & 0 & 0.878102 & -0.318597 & 1.247668 \\ 2 & 6 & 0 & 1.635327 & -0.783226 & 0.000000 \\ 3 & 6 & 0 & 0.878102 & -0.318597 & -1.247668 \\ 4 & 6 & 0 & -0.568901 & -0.886083 & -1.279077 \\ 5 & 6 & 0 & -0.955442 & -1.657448 & 0.000000 \\ 6 & 6 & 0 & -0.568901 & -0.886083 & 1.279077 \\ 7 & 6 & 0 & 0.878102 & 1.245884 & 1.270499 \\ 8 & 6 & 0 & -2.392771 & -2.036083 & 0.000000 \\ 9 & 6 & 0 & 0.309242 & 1.748203 & 0.000000 \\ 10 & 6 & 0 & 0.878102 & 1.245884 & -1.270499 \\ 11 & 7 & 0 & -0.730363 & 2.520450 & 0.000000 \\ 12 & 1 & 0 & 1.416275 & -0.624513 & 2.143007 \\ 13 & 1 & 0 & 2.653570 & -0.389067 & 0.000000 \\ 14 & 1 & 0 & 1.727499 & -1.868303 & 0.000000 \\ 15 & 1 & 0 & 1.416275 & -0.624513 & -2.143007 \\ 16 & 1 & 0 & -1.294078 & -0.080902 & -1.429269 \\ 17 & 1 & 0 & -0.686147 & -1.547069 & -2.136958 \\ 18 & 1 & 0 & -0.364623 & -2.585563 & 0.000000 \\ 19 & 1 & 0 & -1.294078 & -0.080902 & 1.429269 \\ 20 & 1 & 0 & -0.686147 & -1.547069 & 2.136958 \\ 21 & 1 & 0 & 0.337490 & 1.629612 & 2.135471 \\ 22 & 1 & 0 & 1.913834 & 1.590934 & 1.332043 \\ 23 & 1 & 0 & -2.899004 & -2.268577 & -0.926112 \\ 24 & 1 & 0 & -2.899004 & -2.268577 & 0.926112 \\ 25 & 1 & 0 & 0.337490 & 1.629612 & -2.135471 \\ 26 & 1 & 0 & 1.913834 & 1.590934 & -1.332043 \\ 27 & 1 & 0 & -1.153201 & 2.838847 & 0.862911 \\ 28 & 1 & 0 & -1.153201 & 2.838847 & -0.862911\end{array}$

Sum of electronic and zero-point Energies= Sum of electronic and thermal Energies=

Sum of electronic and thermal Enthalpies=

Sum of electronic and thermal Free Energies=
$-445.694426$

$-445.684131$

$-445.683187$

$-445.730175$

$\operatorname{Ama}^{+}(\mathrm{III})$

$\begin{array}{rr}1 & 6 \\ 2 & 6 \\ 3 & 6 \\ 4 & 6 \\ 5 & 6 \\ 6 & 6 \\ 7 & 6 \\ 8 & 6 \\ 9 & 6 \\ 10 & 6 \\ 11 & 7 \\ 12 & 1 \\ 13 & 1 \\ 14 & 1 \\ 15 & 1 \\ 16 & 1 \\ 17 & 1 \\ 18 & 1 \\ 19 & 1 \\ 20 & 1 \\ 21 & 1 \\ 22 & 1 \\ 23 & 1 \\ 24 & 1 \\ 25 & 1 \\ 26 & 1 \\ 27 & 1 \\ 28 & 1\end{array}$

$\begin{array}{rrr}0.923219 & -0.687401 & 1.262057 \\ 1.637589 & -1.195743 & 0.000000 \\ 0.923219 & -0.687401 & -1.262057 \\ -0.547317 & -1.154933 & -1.263904 \\ -1.207910 & -0.715660 & 0.000000 \\ -0.547317 & -1.154933 & 1.263904 \\ 0.923219 & 0.854630 & 1.276496 \\ -2.594709 & -0.193357 & 0.000000 \\ 0.358830 & 1.379799 & 0.000000 \\ 0.923219 & 0.854630 & -1.276496 \\ -0.415579 & 2.442147 & 0.000000 \\ 1.432506 & -1.047956 & 2.154616 \\ 2.679262 & -0.869158 & 0.000000 \\ 1.650442 & -2.286200 & 0.000000 \\ 1.432506 & -1.047956 & -2.154616 \\ -1.072493 & -0.775868 & -2.141394 \\ -0.569959 & -2.249855 & -1.329234 \\ -1.072493 & -0.775868 & 2.141394 \\ -0.569959 & -2.249855 & 1.329234 \\ 0.380399 & 1.243863 & 2.139094 \\ 1.953469 & 1.219584 & 1.347374 \\ 0.380399 & 1.243863 & -2.139094 \\ 1.953469 & 1.219584 & -1.347374 \\ -0.742050 & 2.856458 & 0.860499 \\ -0.742050 & 2.856458 & -0.860499 \\ -2.812381 & 0.398211 & -0.890584 \\ -2.812381 & 0.398211 & 0.890584 \\ -3.311898 & -1.026340 & 0.000000\end{array}$

Sum of electronic and zero-point Energies=

Sum of electronic and thermal Energies=

Sum of electronic and thermal Enthalpies=

Sum of electronic and thermal Free Energies=

$-445.727708$

$-445.717586$

$-445.716642$

$-445.762902$ 


\section{Ada}

$\begin{array}{rrr}0.000000 & 0.000000 & 1.774934 \\ 0.621527 & 0.621527 & 2.425680 \\ -0.621527 & -0.621527 & 2.425680 \\ 0.889683 & -0.889683 & 0.889683 \\ 1.520547 & -1.520547 & 1.520547 \\ -0.889683 & 0.889683 & 0.889683 \\ -1.520547 & 1.520547 & 1.520547 \\ 0.000000 & 1.774934 & 0.000000 \\ 0.621527 & 2.425680 & 0.621527 \\ -0.621527 & 2.425680 & -0.621527 \\ 1.774934 & 0.000000 & 0.000000 \\ 2.425680 & -0.621527 & -0.621527 \\ 2.425680 & 0.621527 & 0.621527 \\ 0.889683 & 0.889683 & -0.889683 \\ 1.520547 & 1.520547 & -1.520547 \\ 0.000000 & 0.000000 & -1.774934 \\ -0.621527 & 0.621527 & -2.425680 \\ 0.621527 & -0.621527 & -2.425680 \\ 0.000000 & -1.774934 & 0.000000 \\ -0.621527 & -2.425680 & 0.621527 \\ 0.621527 & -2.425680 & -0.621527 \\ -1.774934 & 0.000000 & 0.000000 \\ -2.425680 & -0.621527 & 0.621527 \\ -2.425680 & 0.621527 & -0.621527 \\ -0.889683 & -0.889683 & -0.889683 \\ -1.520547 & -1.520547 & -1.520547\end{array}$

Sum of electronic and zero-point Energies= Sum of electronic and thermal Energies= Sum of electronic and thermal Enthalpies= $-390.638558$ $-390.631860$ Sum of electronic and thermal Free Energies

\section{$\operatorname{Ada}^{+}(\mathbf{l})$}

$\begin{array}{rrrrrr}1 & 6 & 0 & -1.264306 & 0.729947 & -1.000887 \\ 2 & 1 & 0 & -1.317997 & 0.760946 & -2.097682 \\ 3 & 1 & 0 & -2.157799 & 1.245806 & -0.647152 \\ 4 & 6 & 0 & 0.000000 & 1.449632 & -0.553782 \\ 5 & 1 & 0 & 0.000000 & 2.497787 & -0.854160 \\ 6 & 6 & 0 & -1.255418 & -0.724816 & -0.553782 \\ 7 & 1 & 0 & -2.163147 & -1.248893 & -0.854160 \\ 8 & 6 & 0 & 0.000000 & -1.459894 & -1.000887 \\ 9 & 1 & 0 & 0.000000 & -1.521892 & -2.097682 \\ 10 & 1 & 0 & 0.000000 & -2.491611 & -0.647152 \\ 11 & 6 & 0 & 1.264306 & 0.729947 & -1.000887 \\ 12 & 1 & 0 & 2.157799 & 1.245806 & -0.647152 \\ 13 & 1 & 0 & 1.317997 & 0.760946 & -2.097682 \\ 14 & 6 & 0 & 1.255418 & -0.724816 & -0.553782 \\ 15 & 1 & 0 & 2.163147 & -1.248893 & -0.854160 \\ 16 & 6 & 0 & 1.260725 & -0.727880 & 1.057314 \\ 17 & 1 & 0 & 1.276133 & -1.761607 & 1.396935 \\ 18 & 1 & 0 & 2.163663 & -0.224360 & 1.396935 \\ 19 & 6 & 0 & 0.000000 & 1.455760 & 1.057314 \\ 20 & 1 & 0 & -0.887530 & 1.985967 & 1.396935 \\ 21 & 1 & 0 & 0.887530 & 1.985967 & 1.396935 \\ 22 & 6 & 0 & -1.260725 & -0.727880 & 1.057314 \\ 23 & 1 & 0 & -2.163663 & -0.224360 & 1.396935 \\ 24 & 1 & 0 & -1.276133 & -1.761607 & 1.396935 \\ 25 & 6 & 0 & 0.000000 & 0.000000 & 1.463488 \\ 26 & 1 & 0 & 0.000000 & 0.000000 & 2.586843\end{array}$

Sum of electronic and zero-point Energies= Sum of electronic and thermal Energies= Sum of electronic and thermal Enthalpies= Sum of electronic and thermal Free Energies=
$-390.314906$ $-390.307097$ $-390.306153$ $-390.345713$ 
$\mathrm{Ada}^{+}$(II)

$\begin{array}{rrrrrr}1 & 6 & 0 & 0.314931 & 0.674387 & 1.251226 \\ 2 & 6 & 0 & 1.022614 & 1.211447 & 0.000000 \\ 3 & 6 & 0 & 0.314931 & 0.674387 & -1.251226 \\ 4 & 6 & 0 & 0.314931 & -0.885594 & -1.279710 \\ 5 & 6 & 0 & 0.918052 & -1.505591 & 0.000000 \\ 6 & 6 & 0 & 0.314931 & -0.885594 & 1.279710 \\ 7 & 6 & 0 & -1.118600 & 1.224650 & 1.279902 \\ 8 & 6 & 0 & 0.824703 & -2.972029 & 0.000000 \\ 9 & 6 & 0 & -1.794836 & 1.313081 & 0.000000 \\ 10 & 6 & 0 & -1.118600 & 1.224650 & -1.279902 \\ 11 & 1 & 0 & 0.823004 & 1.042593 & 2.140454 \\ 12 & 1 & 0 & 1.013091 & 2.304059 & 0.000000 \\ 13 & 1 & 0 & 2.072604 & 0.923393 & 0.000000 \\ 14 & 1 & 0 & 0.823004 & 1.042593 & -2.140454 \\ 15 & 1 & 0 & -0.702534 & -1.264050 & -1.399295 \\ 16 & 1 & 0 & 0.873577 & -1.239934 & -2.144974 \\ 17 & 1 & 0 & 1.993103 & -1.260475 & 0.000000 \\ 18 & 1 & 0 & -0.702534 & -1.264050 & 1.399295 \\ 19 & 1 & 0 & 0.873577 & -1.239934 & 2.144974 \\ 20 & 1 & 0 & -1.771878 & 0.744738 & 2.019722 \\ 21 & 1 & 0 & -1.114069 & 2.278397 & 1.633784 \\ 22 & 1 & 0 & 0.803321 & -3.530263 & -0.925849 \\ 23 & 1 & 0 & 0.803321 & -3.530263 & 0.925849 \\ 24 & 1 & 0 & -1.771878 & 0.744738 & -2.019722 \\ 25 & 1 & 0 & -1.114069 & 2.278397 & -1.633784 \\ 26 & 1 & 0 & -2.859986 & 1.527286 & 0.000000\end{array}$

Sum of electronic and zero-point Energies= Sum of electronic and thermal Energies= Sum of electronic and thermal Enthalpies=

Sum of electronic and thermal Free Energies=
$-390.258696$ $-390.248971$ $-390.248027$ $-390.293923$

\section{$\mathrm{Ada}^{+}(\mathrm{III})$}

$\begin{array}{rrrrrr}1 & 6 & 0 & -0.875727 & -0.441729 & -1.263459 \\ 2 & 6 & 0 & -1.574718 & -0.963874 & 0.000000 \\ 3 & 6 & 0 & -0.875727 & -0.441729 & 1.263459 \\ 4 & 6 & 0 & 0.614068 & -0.864384 & 1.259046 \\ 5 & 6 & 0 & 1.246253 & -0.390926 & 0.000000 \\ 6 & 6 & 0 & 0.614068 & -0.864384 & -1.259046 \\ 7 & 6 & 0 & -0.875727 & 1.110482 & -1.267341 \\ 8 & 6 & 0 & 2.598656 & 0.197483 & 0.000000 \\ 9 & 6 & 0 & -0.260031 & 1.557518 & 0.000000 \\ 10 & 6 & 0 & -0.875727 & 1.110482 & 1.267341 \\ 11 & 1 & 0 & -1.363514 & -0.816966 & -2.161164 \\ 12 & 1 & 0 & -2.619773 & -0.651210 & 0.000000 \\ 13 & 1 & 0 & -1.572755 & -2.055050 & 0.000000 \\ 14 & 1 & 0 & -1.363514 & -0.816966 & 2.161164 \\ 15 & 1 & 0 & 1.134554 & -0.489329 & 2.138747 \\ 16 & 1 & 0 & 0.674932 & -1.958519 & 1.277643 \\ 17 & 1 & 0 & 1.134554 & -0.489329 & -2.138747 \\ 18 & 1 & 0 & 0.674932 & -1.958519 & -1.277643 \\ 19 & 1 & 0 & -0.357442 & 1.505152 & -2.139137 \\ 20 & 1 & 0 & -1.912342 & 1.464409 & -1.306525 \\ 21 & 1 & 0 & -0.357442 & 1.505152 & 2.139137 \\ 22 & 1 & 0 & -1.912342 & 1.464409 & 1.306525 \\ 23 & 1 & 0 & 2.808628 & 0.777933 & 0.896849 \\ 24 & 1 & 0 & 2.808628 & 0.777933 & -0.896849 \\ 25 & 1 & 0 & 3.312028 & -0.642910 & 0.000000 \\ 26 & 1 & 0 & 0.498548 & 2.330189 & 0.000000\end{array}$

Sum of electronic and zero-point Energies= Sum of electronic and thermal Energies=

Sum of electronic and thermal Enthalpies= Sum of electronic and thermal Free Energies=
$-390.316951$ $-390.308028$ $-390.307084$ $-390.350448$ 